\title{
Adenocarcinoma of the Appendix
}

\author{
Moussa Sylla1, Pierlesky Elion Ossibi', Somuah Tenkorang², Ismael Dandakoye1, \\ KarimIbn Majdoub' ${ }^{1}$ Imane Toughrai', Said Ait Laalim', Khalid Mazaz ${ }^{1}$
}

${ }^{1}$ Department of Visceral Surgery B, Hassan II University Hospital, Fez, Morocco

${ }^{2}$ Departement of Urology, Hassan II University Hospital, Fez, Morocco

Email: moussa.sylla@usmba.ac.ma

How to cite this paper: Sylla, M., Ossibi, P.E., Tenkorang, S., Dandakoye, I., Majdoub, K., Toughrai, I., Laalim, S.A. and Mazaz, K. (2018) Adenocarcinoma of the Appendix. Surgical Science, 9, 115-121. https://doi.org/10.4236/ss.2018.93012

Received: June 24, 2017

Accepted: March 19, 2018

Published: March 22, 2018

Copyright $\odot 2018$ by authors and Scientific Research Publishing Inc. This work is licensed under the Creative Commons Attribution International License (CC BY 4.0).

http://creativecommons.org/licenses/by/4.0/

\begin{abstract}
Objective: To report the experience of our department on adenocarcinoma of the appendix through a series of six (6) cases. Materials and Methods: This is a retrospective study carried out from January 2010 to June 2015 collating all cases of adenocarcinoma of the appendix diagnosed at the department of visceral surgery B in Hassan II University Hospital in Fez. Results: Six cases of adenocarcinoma of the appendix were recorded during the determined period of study. The mean age was $54.67 \pm 16.99$ years with the extreme ages ranging from 28 to 78 years. There was a female predominance with a sex ratio of 2 . All patients had no specific medical history. The revealing symptoms were the following: appendicitis syndrome (1 case), bowel obstruction (1 case), psoas abscess ( 1 case) and fortuitous discovery during laparotomy for another problem ( 3 cases). The following histological types found were: colloid adenocarcinoma (3 cases), mucinous adenocarcinoma ( 2 case), mucinous cystadenocarcinoma associated with a moderately differentiated adenocarcinoma of the appendix ( 1 case). The surgical procedure consisted of a right hemicolectomy in 3 patients. Appendectomy followed by a right hemicolectomy and drainage of psoas abscess was performed in two patients. Palliative ileocecal resection and Bouilly-Volkmann ileocolostomy was performed in one patent who had carcinosis. All patients received adjuvant chemotherapy. The following occurred during follow-up: carcinosis (2 patients), a locoregional recurrence ( 2 patients). Two patients never returned for follow-up. Conclusion: Tumor of the appendix is a rare and exceptional pathology. These tumors are generally discovered fortuitously or during histology of appendectomy specimens. The treatment is usually surgical.
\end{abstract}

\section{Keywords}

Adenocarcinoma, Appendix 


\section{Introduction}

The appendix is a unique organ whose origin and function are not absolutely clear. This is probably why different types of tumors arise from its cells [1]. The malignant tumors of the appendix are very rare. A study of primary malignant tumors in the United States found an incidence of 0.12 cases per 1,000,000 inhabitants per year. A Hong Kong series of 1492 appendectomies reported 17 cancers of which 3 were adenocarcinomas [2]. They are often discovered fortuitously either on specimens of appendectomy or during a laparotomy for another pathology. Through this series of six clinical cases, we will discuss the difficulties surrounding the diagnosis and the management of adenocarcinoma of the appendix.

\section{Materials and Methods}

This is a retrospective study carried out from January 2010 to June 2015 in the department of visceral surgery B of the Hassan II University Hospital Center in Fes, Morocco.

Patients followed or hospitalized for adenocarcinoma of the appendix were included in our study.

Benign or inflammatory tumors of the appendix have been excluded.

Our patients are registered in a computer system of the hospital allowing us to access their medical records and contact them as often as necessary.

Epidemiologic factors analyzed included patient age at diagnosis, gender, presenting symptoms or circumstances of discovery, medical history, preoperative imaging, intraoperative findings, operation performed, pathology results, use of postoperative chemotherapy, and patients outcomes. Patients were classified according to the type of preoperative imaging and whether the imaging was able to detect the presence of a neoplasm preoperatively or not. Intraoperative values recorded including the presence of metastatic disease, whether a neoplasm was suspected during the initial operation. The operation performed was initial operation or a completion operation. Patient survival, follow up for a minimum of 3 years, and complications were also noted.

\section{Course of the Study}

After obtaining permission from the local ethics committee, we started the selection of patients from the registry data followed by the exploitation of medical records in the department of visceral surgery B of the Hassan II University Hospital Center of Fez in Morocco.

\section{Statistical Analysis}

The data collected was entered and analyzed on Excel 2010.

\section{Results}

During the study period, we recorded 6 cases of adenocarcinoma of the appen- 
dix. The mean age was $54.67 \pm 16.99$ years with extreme ages ranging from 28 to 78 years. There was a female sex predominance with a sex ratio of 2 . All patients had no specific medical history. The revealing symptoms were the following: appendicitis syndrome ( 1 case), bowel occlusion ( 1 case), psoas abscess ( 1 case) and fortuitous discovery during laparotomy for another pathology (3 cases).

Abdominal ultrasound was performed in a patient who presented appendicitis syndrome. It showed a simple appendicitis with the appendix measuring $12 \mathrm{~mm}$ in diameter.

Five patients had preoperative abdominal computed tomography scan that objectified a collection in the right iliac fossa measuring $63 \times 68 \times 90 \mathrm{~mm}$ which was in contact with the right colon and ipsilateral Psoas muscle. It is shown in Figure 1. This collection was found to be probably originating from the appendix. One patient had a large hypodense collection $(12 \mathrm{~cm}$ in height) in the right iliac fossa extending to the ipsilateral Psoas iliacus muscle as well as on the right anterolateral abdominal wall. A lateral uterine mass was found in a patient; it was an irregular polylobed mass in the right iliac fossa with a necrotized center narrowing the digestive lumen measuring $121 \times 69 \mathrm{~mm}$. Another patient had dilated loops of small bowel distension proximal to an irregular and circumferential thickening of the last segment of the ileum. The CT scan of a patient who underwent appendectomy was normal.

The following histological types were found: colloid adenocarcinoma (3 cases), mucinous adenocarcinoma (2 case), mucinous cystadenocarcinoma associated with a moderately differentiated adenocarcinoma of the appendix (1 case).

Surgery was performed on all the patients. Surgical exploration in one patient found a gelatinous collection attached to the tip of the appendix which was thick

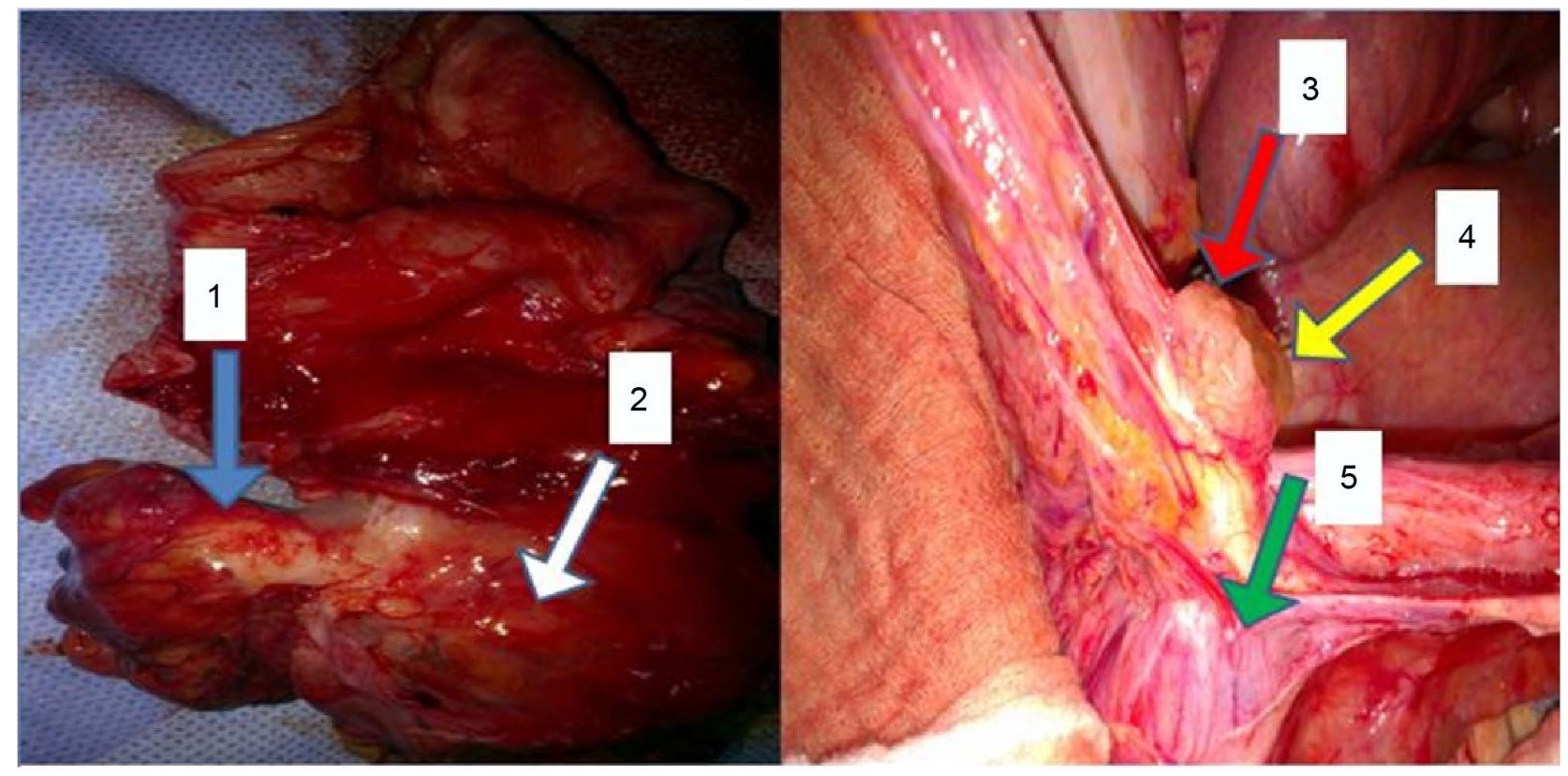

Figure 1. Preoperative images: 1-Posterior view of the appendix; 2-Posterior View of the caecum; 3-anterior view of the appendix; 4-Gelatinous collection attached to the tip of the appendix; 5-anterior view of the caecum. 
and infiltrated in its entirety as well as the cecum. Two patients had a thickened appendix and cecum; a deep thickened cecal pouch with an appendix in the internal lateral-cecal position. For the fourth patient, the tip of the appendix was implanted within the Psoas muscle, the release of the appendix revealed the presence of mucus in retroperitoneal cavity. The presence of a tumor of the appendix extending to the caecum was found in another patient. For the last one, the appendix was phlegmonous, perforated at its point with the presence of gelatinous fluid. There was an extensive carcinosis in the mesentery and parietal peritoneum.

A right hemicolectomy was performed in 3 patients; two patients had appendectomy followed by right hemicolectomy and drainage of psoas abscess. Palliative ileocecal resection and Bouilly Volkmann ileocolostomy was performed in the patient with carcinosis.

All patients received adjuvant chemotherapy. During follow up, carcinosis occurred in two patients and a locoregional recurrence in 2 other patients (Figure 2). Two patients never returned for follow-up.

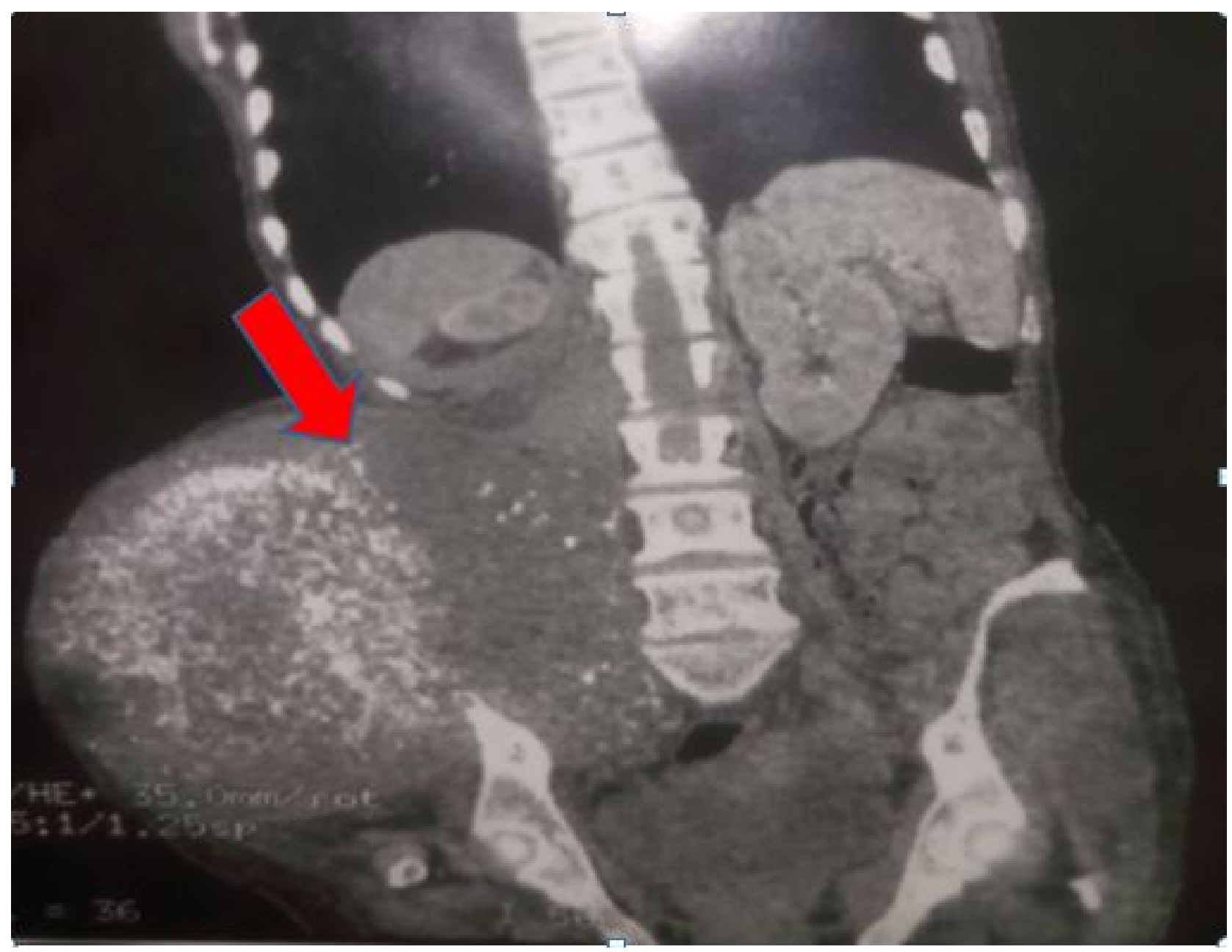

Figure 2. Image of tumor recurrence (the arrow shows the tumor recurrence with invasion of the right iliac bone and anterolateral abdominal wall). 


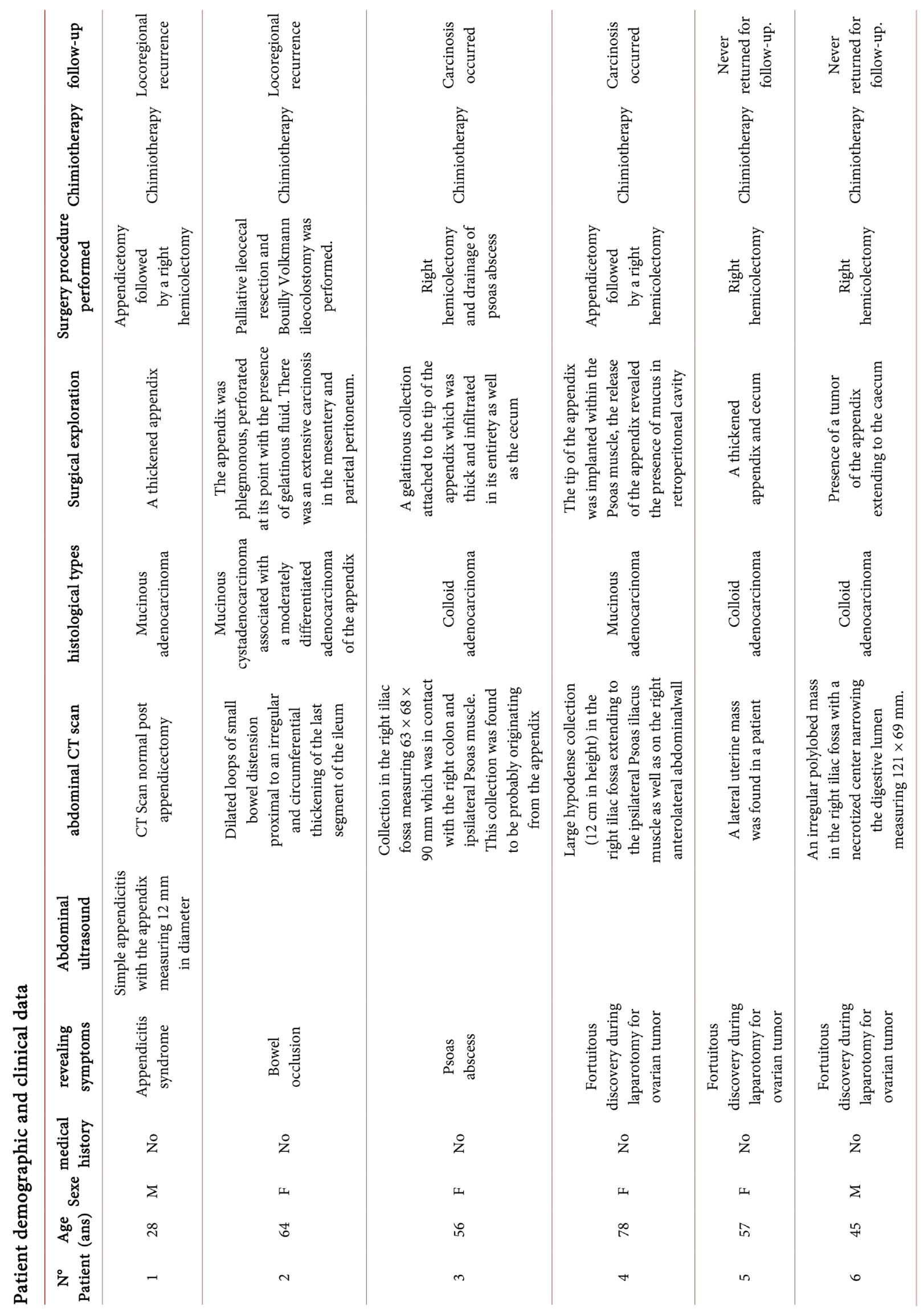

DOI: $10.4236 /$ ss.2018.93012 


\section{Discussion}

The Cancer of the appendix is very rare and is typically found incidentally in approximately $1 \%$ of appendectomies. Appendiceal neoplasms account for approximately $0.4 \%$ of gastrointestinal tumors [3]. Among adenocarcinomas of the appendix, there are three subtypes: mucinous (55\%), colloid (34\%) and carcinoid tumors (11\%) with mixed morphology. The average age of diagnosis is during the fifth decade of life, with an equal male to female ratio for all the subtypes except colloid adenocarcinomas which may have a higher incidence in men [2].

Non-carcinoid Primary tumors of the appendix (colloid and mucinous) are rare with less than 300 cases reported in the literature. A surgeon is unlikely to encounter more than one case in his career, so it is necessary to learn about its natural history through experiences from specialized centers [4].

The cases of tumors of the appendix are diagnosed after appendicectomy by histological analysis and rarely give any symptoms and signs either by clinical examination or by hematological indices [5].

The surgical treatment is poorly codified, it depends on the locoregional extension of the lesion, the size of the tumor and the histological type that contribute to the selection of the surgical treatment and all must be estimated by the surgeon to determine the final choice.

Appendicectomy is usually the only treatment required for an appendicular carcinoid tumor less than $1.5 \mathrm{~cm}$.

Right hemicolectomy is indicated for carcinoid tumors larger than $2 \mathrm{~cm}$ in diameter or for the non-carcinoid appendix cancer.

For advanced appendix cancer, a reduction surgery (cytoreduction) may be performed. Sometimes a reduction surgery will be followed by chemotherapy to destroy the remaining cancer cells [6].

The role for adjuvant chemotherapy is controversial and there are no controlled data regarding the efficacy of chemotherapy following resection of localized disease. The role of adjuvant radiotherapy is also unclear due to the lack of randomized data [7].

Malignant tumors of the appendix represent a diagnostic and therapeutic challenge. No standard of care is established due to the rare occurrence of this pathology. Some studies have shown that there is a clear survival benefit to the addition of a hemicolectomy. Our study and review of the literature are a summary of tumor of the appendix, with management based more on the personal opinion of oncologists who treat this type of tumor. Prognosis of adenocarcinoma depends on the subtype and extent of disease. Those patients would have a worse prognosis when intraperitoneal seeding occurred. Mucinous adenocarcinoma has a more favorable prognosis because it does not exhibit hematogenous or lymphatic spread.

\section{Conclusions}

The rare occurrence of adenocarcinoma of the appendix as well as the lack of 
specific clinical symptoms, likely explains the delay in establishing the diagnosis for this pathology. Complete surgical excision and/or chemotherapy can sometimes provide a prolonged survival rate.

The accidental discovery of these tumors with the necessity of a second-stage surgery remains the main difficulty in the management of these tumors. The surgeon is sometimes in front of a hemicolectomy based only on the intraoperative macroscopic appearance of the appendix without histological evidence.

\section{References}

[1] Alvarez-Laso, C., Azcano, E., Carrion, J.F., Rodríguez, D. and Riera, J.R. (2017) Goblet Cell Carcinoid Tumors of the Appendix: A Report of Two Cases, Review of a Rare Clinical Entity, and Guidelines for Treatment. Open Access Library Journal, 4, e3256. https://doi.org/10.4236/oalib.1103256

[2] McCusker, M.E., Coté, T.R., Clegg, L.X. and Sobin, L.H. (2002) Primary Malignant Neoplasms of the Appendix: A Population-Based Study from the Surveillance, Epidemiology and End-Results Program, 1973-1998. American Cancer Society, 94, 3307-3312. https://doi.org/10.1002/cncr.10589

[3] Ruoff, C., Hanna, L., Zhi, W.Q., Shahzad, G., Gotlieb, V. and Saif, M.W. (2011) Cancers of the Appendix: Review of the Literatures. ISRN Oncology, 2011, Article ID: 728579. https://doi.org/10.5402/2011/728579

[4] Hartley, J.E., Drew, P.J., Qureshi, A., MacDonald, A. and Monson, J.R.T. (1996) Primary Adenocarcinoma of the Appendix. Journal of the Royal Society of Medici, 89, 111-113. https://doi.org/10.1177/014107689608900220

[5] Alexiou, K., Ioannidis, A., Drikos, I., Karanikas, I., Fotopoulos, A., Chorti, M. and Economou, N. (2014) Adenocarcinoma of the Appendix in Young Men with Acute Appendicitis. Surgical Science, 5, 206-209. https://doi.org/10.4236/ss.2014.55035

[6] Englehardt, R.K., Durrani, N.K. and Mittal, V.K. (2010) Management and Outcomes in Primary Tumors of the Appendix. Journal of Cancer Therapy, 1, 174-180. https://doi.org/10.4236/jct.2010.14027

[7] Powell, E.D., Macdonald, D.B., Elkeilani, A.M. and Asmis, T.R. (2009) A Case of Appendiceal Adenocarcinoma with Clinical Benefit from FOLFOX and Bevacizumab. Case Reports in Oncology, 2, 111-115. https://doi.org/10.1159/000229245 